Questions vives

\section{Questions Vives}

Recherches en éducation

$N^{\circ} 25$ | 2016

L'activité des enseignants face au décrochage scolaire : Quelles difficultés et quelles mises en œuvre professionnelles?

\title{
Risque de décrochage après une rupture de contrat d'apprentissage : l'exemple d'un CFA en Alsace
}

Risk of dropout after a breach of apprenticeship contract: the example of an apprentice training center in Alsace, France

Stéphane Guillon et Stéphanie Hinsinger

\section{(2) OpenEdition}

Journals

Édition électronique

URL : http://journals.openedition.org/questionsvives/1928

DOI : $10.4000 /$ questionsvives. 1928

ISSN : $1775-433 \mathrm{X}$

Éditeur

Université Aix-Marseille (AMU)

Édition imprimée

Date de publication : 7 juillet 2016

ISBN : 978-2-912643-49-0

ISSN : 1635-4079

Référence électronique

Stéphane Guillon et Stéphanie Hinsinger, «Risque de décrochage après une rupture de contrat d'apprentissage : l'exemple d'un CFA en Alsace », Questions Vives [En ligne], N 25 | 2016, mis en ligne le 05 septembre 2016, consulté le 01 mai 2019. URL : http://journals.openedition.org/

questionsvives/1928; DOI : 10.4000/questionsvives.1928

Ce document a été généré automatiquement le 1 mai 2019.

\section{cc) (†)}

Questions Vives est mis à disposition selon les termes de la licence Creative Commons Attribution -

Pas d'Utilisation Commerciale - Pas de Modification 4.0 International. 


\title{
Risque de décrochage après une rupture de contrat d'apprentissage : l'exemple d'un CFA en Alsace
}

\author{
Risk of dropout after a breach of apprenticeship contract: the example of an \\ apprentice training center in Alsace, France
}

Stéphane Guillon et Stéphanie Hinsinger

\section{Introduction}

1 La rupture de contrat dans le cadre d'un apprentissage, même si elle reste peu fréquente, est un événement susceptible de détériorer la stabilité du parcours de formation de l'apprenti. Dans le cas de l'alternance, les effets sur les trajectoires juvéniles de l'expérience de rupture du lien salarial sont d'abord corrélés à la problématique de l'absence de diplomation, qui est l'un des indicateurs du décrochage scolaire chez les jeunes.

2 Régulièrement annoncé comme la panacée de lutte contre le chômage juvénile (Arrighi \& Brochier, 2005), tout en suscitant chez certains auteurs la crainte d'une forme d'« obnubilation entrepreneuriale» (Longhi, 2011, p. 181), le contrat d'apprentissage est un environnement particulier dans le cadre d'une réflexion sur le décrochage scolaire, dans la mesure où il n'échappe ni aux conditions classiques de la scolarisation ni au risque économique d'une rupture du lien salarial. Plusieurs questions peuvent alors être posées. La rupture du contrat d'apprentissage augure-t-elle un décrochage chez l'apprenti, décrochage entendu dans le sens restreint d'absence de diplomation à la fin du parcours de formation en alternance? Quelles sont les conséquences des ruptures de contrat d'apprentissage dans le parcours de formation des jeunes apprentis? Et quels sont les contextes dans lesquels la rupture ne conduit pas à l'échec?

3 Si l'on adopte le point de vue du système éducatif, ce type de rupture n'est pas simplement une interruption du lien salarial : il se double d'une interruption du parcours 
de formation et peut présenter un risque à la fois de non-diplomation du jeune en fin de parcours, mais également de détérioration de son employabilité dans le sens où le décrochage déstabilise a posteriori, toutes choses égales par ailleurs, la qualité de la primoinsertion. L'avenir est également obéré en termes de poursuite d'études : «Un retour sur la connaissance actuelle des caractéristiques des ruptures invite à faire évoluer la problématique pour la centrer sur l'analyse de ce qui se passe après la résiliation du contrat d'apprentissage » (Cart, Ducourant, Henguelle, Surelle \& Toutin, 2007, p. 15). Dès le moment où il entre en apprentissage, le jeune se projette dans une trajectoire professionnelle de formation. La rupture du contrat d'apprentissage est alors pour lui synonyme d'instabilité ; elle ébranle en tout cas son projet de formation. Cette cassure, lorsqu'elle intervient, est souvent multifactorielle et doit être analysée d'une manière globale comme un processus au cours duquel différents acteurs entrent en jeu: l'apprenti-rompant et sa famille, l'entreprise d'accueil, le maître d'apprentissage, les chambres consulaires (dans leur rapport administratif avec l'entreprise) et le centre de formation (dans son rapport formatif avec l'apprenti) forment une relation tripartite au cœur de la compréhension du phénomène de rupture, et parfois de décrochage, que nous tenterons de décrire.

Notre article cherche à comprendre les raisons pour lesquelles certains apprentisrompants, malgré l'expérience de la rupture du contrat d'apprentissage, parviennent à obtenir leur diplôme en bout de course, pendant que d'autres décrochent en chemin. $\mathrm{Ce}$ phénomène de la rupture contractuelle dans le cadre de l'alternance peut être étudié par une démarche heuristique à la fois synchroniquement (le moment de l'alternance est appréhendé comme une socialisation professionnelle dont les dimensions sont à interroger), mais également longitudinalement dans la mesure où les événements ayant lieu pendant le contrat d'alternance (décrochage, abandon, réorientation, diplomation) peuvent trouver leur origine en amont de la signature du contrat avec l'employeur, au moment de la construction d'un projet plus ou moins contraint, plus ou moins documenté. C'est cette double approche que nous adopterons.

\section{Cadre théorique}

5 Notre réflexion puise dans un cadre théorique riche de travaux portant à la fois sur le décrochage juvénile (1), l'alternance comme modalité de formation (2), la rupture du contrat d'apprentissage (3) et les mesures préventives face au décrochage et à la rupture contractuelle (4).

6 1. Nous utiliserons la définition du décrochage telle qu'elle apparait dans le Code de l'Éducation (art. L313-7) qui traite des jeunes n'ayant pas atteint un niveau de qualification minimum, niveau qui est défini par le décret $n^{\circ}$ 2010-1781 du 31 décembre 2010. En ce sens, les jeunes "décrocheurs" sont ceux qui ont quitté un cursus de formation de l'enseignement secondaire sans obtenir le diplôme visé par cette formation : nous considérerons ici comme décrocheurs les apprentis, quel que soit le niveau de diplôme préparé, qui n'ont pas atteint la diplomation en fin de parcours.

7 Le décrochage scolaire reste une priorité pour les pouvoirs publics. Le contrat d'apprentissage, voie de formation par l'alternance de la formation initiale, même s'il peut apparaître comme un dispositif favorable à l'insertion professionnelle dans la mesure où il rapproche le système éducatif et le monde de l'entreprise, n'évite pas le risque de décrochage. Cart, Toutin-Trelcat, Henguelle (2010) rappellent néanmoins 
combien la réalité du décrochage dans le cadre de l'apprentissage reste difficile à évaluer quantitativement, dans la mesure où les données sont fragmentées et éparpillées. Pour Geay (1998), "les élèves qui "décrochent" présentent la motivation intrinsèque la plus faible, liée à la baisse de la compétence perçue et l'amotivation la plus élevée. La motivation intrinsèque avec ses composantes semble bien déterminer la persévérance dans une activité. » Les données du Céreq (2011), récoltées dans le cadre de l'enquête "Génération 2007-2010 ", sont les plus exhaustives concernant les décrocheurs: elles donnent à connaître l'évolution des situations professionnelles des sortants du système éducatif pendant leurs trois premières années de vie active et présentent, entre autres, des résultats comparatifs entre le devenir des apprentis ayant été au terme de leur contrat et celui des apprentis l'ayant rompu.

8 2. L'alternance, entendue comme un ensemble de dispositifs de formation régis par le Code de l'Éducation et par le Code du Travail, a été peu investie par la recherche. Pour Tanguy (2005), les travaux universitaires ont focalisé leur attention sur le centre (l'enseignement général) et non sur les limites (la formation professionnelle), si bien que les questions liées à l'alternance et à l'apprentissage ont longtemps fait figure de parents pauvres de la recherche universitaire, ce que confirme Moreau : «Lointain petit canton rural oublié de la formation professionnelle, l'apprentissage en entreprise n'en a été que plus invisible» (2008, p. 121). Dans une approche qui privilégie l'analyse historique du système éducatif et des dispositifs liés à la formation professionnelle, les travaux sur l'apprentissage ont d'abord montré que l'évolution de l'apprentissage comme dispositif alternant formation et salariat devait se comprendre dans un système d'opposition entre voie scolaire et alternance, même si le législateur souhaitait que les deux filières restent "complémentaires et non concurrentielles ». Pour la période moderne, après un renforcement de la place de l'école dans la formation professionnelle, on assiste à un retour de la logique professionnaliste, au détriment de la voie scolaire. Kergoat et Capdevielle-Mougnibas datent ainsi la première transformation de l'apprentissage des années 1970 de la loi fondatrice du 16 juillet $1971^{1}$ qui alignait l'apprentissage sur les formes scolaires: "L'État décide [...] d'étendre la scolarisation de la formation professionnelle initiale à l'apprentissage. » (2013, p. 6). Après 1987 (la réforme Seguin ${ }^{2}$ ), une seconde phase de scolarisation de la formation professionnelle a " aspiré » l'ensemble du dispositif vers le haut " (Arrighi \& Brochier, 2005), renforçant les effets des héritages sociaux et scolaires. C'est la crise du chômage (y compris juvénile) qui a inversé le processus et favorisé la voie professionnelle par l'alternance salariée : face à la hausse structurelle du chômage, l'Éducation nationale a par ailleurs intensifié ses liens avec le monde économique, multiplié les stages d'immersion et tous les dispositifs de mise en contact des jeunes avec le monde économique, de plus en plus tôt. En même temps que se précarisaient les trajectoires juvéniles (Rose, 1998 ; Baudelot \& Establet, 2000 ; Mauger, 2001 ; Boléguin, 2011), l'apprentissage salarié était relancé. Ce processus s'inscrivait dans le mouvement général de décentralisation (surtout la loi quinquennale du 20 décembre 1993) qui a participé du renforcement du poids des acteurs économiques dans la construction de l'offre d'apprentissage et sa mise en œuvre. Tanguy (2013) souligne le fait que ce processus, en confiant la formation professionnelle initiale aux Régions, a eu tendance à "externaliser l'apprentissage hors du système éducatif ». Surtout la loi de programmation pour la cohésion sociale du 18 janvier 2005 a modifié le statut des apprentis et renforcé la formation des formateurs en Centre de Formation d'Apprentis (CFA), intensifiant encore les relations entre apprenti, maitre d'apprentissage et formateurs. La logique entrepreneuriale et la présence du monde économique dans 
l'arsenal scolaire de lutte contre le décrochage, la non-qualification et le chômage juvéniles ont ainsi été renforcées. La mise au travail du groupe juvénile s'inscrit dans un discours et une mise en application postulant que la remédiation scolaire ne peut plus passer massivement par une recherche de rescolarisation.

Néanmoins, le contrat en alternance, lorsqu'il est rompu, s'apparente à une rupture scolaire, dans la mesure où, dans le cas du contrat d'apprentissage, il reste un dispositif de formation initiale. L'alternance continue d'apparaître comme un remède à l'échec scolaire et au décrochage, mais également comme un moyen d'insertion professionnelle pour les jeunes chômeurs et non pas comme un mode spécifique d'apprentissage valable pour tout public (Geay, 1998), ce qui peut avoir pour effet de détériorer l'image de cette filière aux yeux des familles, des élèves et des acteurs du système scolaire. Pour l'auteur, l'alternance est une mise en relation de deux systèmes aux logiques contradictoires, à la fois dans le rapport aux savoirs et dans les stratégies d'apprentissage. De son côté, Moreau souligne que l'entrée en apprentissage est liée à l'articulation entre «l'amour d'un métier» et le "désamour pour l'école» (2003, p. 150), questionnant la problématique d'orientation scolaire où une nouvelle fois l'alternance apparait comme la première réponse institutionnelle face au risque de décrochage.

L'apprentissage est également questionné en tant que dispositif spécifique de formation. La loi du 2 juillet 1980 définissant juridiquement l'alternance induit deux dispositions : un apprentissage qui fait de l'entreprise le lieu central des acquisitions et le travail comme activité au service de la formation. Le principe majeur de la pédagogie de l'alternance réside dans l'utilisation de l'expérience et des acquis professionnels pour donner du sens à une formation plus théorique dispensée dans les centres de formation. Il s'agit donc d'une démarche inductive impliquant une interaction entre l'entreprise et l'organisme assurant la formation théorique et pratique. Cette démarche d'apprentissage peut apparaitre comme une alternative attractive pour les jeunes qui recherchent une expérience du monde du travail.

11 Dans un contexte de difficulté d'insertion des jeunes, les politiques publiques encouragent ainsi à rénover dans ce sens l'ensemble des systèmes de formation. L'enjeu central de l'alternance est l'insertion professionnelle qui remet en cause la formation scolaire jugée par trop théorique. L'alternance ne serait efficace pourtant qu'à certaines conditions (Moreau, 2002), dans la mesure où la formation par alternance participe d'une construction identitaire professionnelle et plus particulièrement d'une socialisation professionnelle (Masdonati, 2007): l'apprenti évolue dans un cadre formatif, mais également dans une organisation productive, une position duale qui peut être une source de cessation de contrat car «la transition école-monde professionnel peut être dangereuse pour un adolescent» (Lamamra \& Masdonati, 2008, p. 69). Cette dualité intervient d'ailleurs dans ce moment particulier du développement personnel qu'est l'adolescence :

« La tâche développementale centrale de l'adolescence est la recherche identitaire, tant personnelle que sociale. [...] Dans le même temps, le moment particulier où intervient la formation professionnelle est susceptible d'amener les jeunes à expérimenter des conflits identitaires entre des sphères d'investissement véhiculant des valeurs différentes, voire opposées. » (Rochat \& Lamamra, 2004, p. 21).

12 Pour les formations en apprentissage, il existerait une socialisation particulière par la famille. Moreau (2003, p. 167) définit ainsi 
«trois formes d'adhésion à l'apprentissage aux frontières plus ou moins franches. Une pré-socialisation dans les familles d'indépendante: milieu favorable à la formation par l'apprentissage, une gestion fine de leurs formations, la transmission des codes et valeurs liés à ce mode de formation ; l'anti-intellectualisme populaire : un désamour de l'école, une dépréciation de la théorie au profit de la pratique et une survalorisation de l'expérience ; un statut d'apprenti qui permet de poursuivre sa formation en se soustrayant aux impératifs d'une scolarité prolongée et incertaine. " sa posture et sa motivation durant son parcours de formation. L'auteur décrit un « apprenti polymorphe » et met en évidence la dualité de la posture du statut d'apprenti salarié et de sa qualité de "jeune». Les difficultés personnelles que l'apprenti va rencontrer durant sa formation impacteraient sa formation du fait de son jeune âge (17 ans en moyenne) (Masdonati, 2007). Dans ce cas, l'apprenti se trouverait face à deux postures possibles : soit il laisserait les difficultés de la sphère privée envahir la sphère professionnelle ; soit il mettrait en place une stratégie pour faire face à ces difficultés et éviter un débordement de celles-ci sur le milieu professionnel (Lamamra \& Masdonati, 2008). L'apprenti se maintiendrait dans une bonne relation à sa formation s'il trouve, au sein de l'entreprise, des conditions satisfaisantes de travail et de relations professionnelles. est appréhendé sous deux angles: d'abord l'engagement en formation qui mène de la première expression d'intention jusqu'au démarrage réel de l'action de formation, ensuite la poursuite de l'engagement à travers la participation effective et les évolutions du processus motivationnel en cours de formation. La motivation est ainsi un processus dynamique :

«L'apprenant devra, au fur et à mesure de son développement, construire des représentations de ce que l'avenir sera pour lui. Cette caractéristique est importante en formation et notamment pour les pré-apprentis qui peuvent se représenter en situation de réussite professionnelle en signant un contrat d'apprentissage et en travaillant dans le cadre de l'apprentissage. » (Carré, 1999, p. 287). d'aubaine chez les employeurs (Cart et al., 2010) ${ }^{3}$.

3. La rupture reste une notion floue: elle est une caractéristique de l'ensemble des contrats de travail et son contenu juridique diffère de son approche en termes de formation professionnelle et d'évaluation de l'apprentissage :

«Le taux de rupture désigne la part des contrats d'apprentissage n'arrivant pas à leur terme. On peut considérer qu'un contrat d'apprentissage rompu et suivi d'un autre contrat dans une période rapprochée (moins de trois mois) ne constitue pas une réelle rupture » (Bentabet, Cart, Henguelle \& Toutin, 2012).

Michaud (2001) identifie la rupture du contrat d'apprentissage comme un processus et définit trois temps d'intervention auprès des apprentis : avant la rupture, au moment de la rupture et après la rupture. La rupture du contrat pour un apprenti, un jeune souvent âgé de moins de 20 ans au parcours scolaire antérieur parfois fragilisé, impacterait négativement l'estime de soi, jusqu'au sentiment de souffrance (Lamamra \& Masdonati, 
2008). Pour Arrighi et Joseph (2005), la rupture du contrat d'apprentissage serait un espace de conflit entre l'apprenti-rompant et son employeur, entre l'apprenti-rompant et son projet de formation professionnelle, mais également entre les organismes de formation et le monde de l'entreprise, un espace de conflictualité qui peut être explicité.

Selon Cart et al. (2007 ; 2008), le premier motif de la rupture est un environnement de travail de mauvaise qualité : conditions de travail non formatrice, inadéquation avec la formation suivie, détournement du contrat d'apprentissage par l'entreprise (heures supplémentaires, tâches non conformes) et le ressenti d'un non-respect de la personne dans son travail. Pour Moreau (2003), il existe « une relation dialectique entre l'ambiance et les conditions de travail, la première fonctionnant comme un antidote fondamental aux secondes ».

Une plongée dans la littérature portant sur les ruptures de contrat d'apprentissage nous donne quelques pistes de compréhension, malgré des données fragmentaires, partielles et des suivis nationaux et régionaux épars et irréguliers. L'enquête Génération 2007-2010 produite par le Centre d'études et de recherches sur les qualifications (Céreq, 2011) sur un échantillon représentatif de 800 apprentis-rompants, tous niveaux confondus, montre que, depuis une vingtaine d'années, plus d'un contrat d'apprentissage sur quatre est rompu (Dréano, 2002 ; Abriac, Rathelot \& Sanchez, 2009 ; Cart et al., 2007) et ces ruptures n'interviennent pas uniquement pendant la période d'essai (les deux premiers mois), mais tout au long du cursus du jeune. Dès les années 1990, les auteurs ont cherché à comprendre les causes de ces ruptures et s'accordent à dire qu'elles sont marquées par des tendances structurelles (Ramé \& Ramé, 1995 ; Moreau, 2003) : plus le niveau augmente, plus le risque de rupture s'éloigne ; les entreprises de petite taille sont le plus concernées (de 1 à 9 salariés) et certains secteurs plus propices à la rupture : l'hôtellerie-restauration, les métiers de bouche, le bâtiment, la coiffure et le commerce. Masdonati et Lamamra (2007) ont également travaillé sur les trajectoires des jeunes apprentis en rupture de contrat, mais en se focalisant sur l'appréciation que les apprentis-rompants portent sur leur rupture et leur parcours ultérieur : les moments critiques du processus de rupture seraient l'orientation et le choix de l'entreprise, l'accueil dans l'entreprise et la fin de la période d'essai. "La rupture résulte toujours d'une conjonction entre des conditions objectives relatives au dispositif de formation et des facteurs subjectifs qui concernent l'ensemble des acteurs, entreprises, apprentis et formateurs. Elle présente ainsi toujours un caractère plurifactoriel.» (Efigip, 2010, p. 22). Les causes de ruptures sont ainsi comprises d'abord dans leur chronologie: en amont, la problématique du choix par défaut ou par proximité géographique, le décalage entre la représentation du métier et la réalité professionnelle, une trop forte exigence de l'employeur, des conditions de travail " non formatrices », un sentiment de discrimination liée au genre, une rémunération trop faible, le manque de savoir-être du jeune, les allergies, la trésorerie, la mobilité géographique.

21 Une autre dimension du phénomène de rupture qui est soulignée est l'importance de la "temporalité de la rupture »: ainsi, les ruptures situées après la période d'essai «sont jugées les plus problématiques, les plus déstabilisantes pour le jeune comme pour l'entreprise et le CFA» (Efigip, 2010, p. 30) .

4. Un consensus se dégage autour de la prise en compte de la rupture du contrat d'apprentissage comme processus qui rendrait centrales les actions de préparation et d'accompagnement des formations en alternance pour lutter contre le décrochage. D'autant plus que l'apprenti en situation de décrochage suite à une rupture de contrat 
court le risque, dans le cas d'un conflit avec son ancien employeur, de détériorer le réseau professionnel qu'il utilise pour prospecter des opportunités d'emploi : «Les ruptures de contrat d'apprentissage sont fréquentes et réduisent les chances d'insertion sur le marché du travail. Pour sécuriser les parcours des apprentis, les accompagner dès leur entrée en formation, dans une logique préventive, semble être une solution efficace » (Bourdon, Guégnard \& Michot, 2012, p. 1).

L'impact d'un dispositif central dans la lutte contre le décrochage et la rupture en cours d'alternance, le Dispositif d'Initiation aux Métiers en Alternance (DIMA) ${ }^{5}$, a été fréquemment souligné : il participerait à la stabilisation des trajectoires des alternants et améliorerait leurs chances de diplomation. Un élève voulant entrer en apprentissage peut demander à accéder à ce "dispositif d'initiation aux métiers en alternance ». Cette formation lui permet de commencer une activité de type professionnel tout en demeurant sous statut scolaire et améliore en amont la connaissance du champ professionnel et du métier (Rongé, 2011). Ce dispositif récent, décrit dans la section 8 du chapitre VII du Livre III du Code de l'Éducation comme étant « l'ensemble des formations en alternance, sous statut scolaire, en centre de formation d'apprentis ", s'inscrit dans un arsenal global de préparation à l'alternance comme une préconisation pour les jeunes en risque de décrochage dans le cursus scolaire classique. L'élève peut ainsi suivre un stage allant de huit à 18 semaines, suivi par un tuteur. L'approche est préventive et non pas curative dans la mesure où elle s'inscrit en amont du risque de décrochage. Pour les élèves de moins de 16 ans, les établissements d'enseignement scolaire peuvent " organiser des visites d'information, des séquences d'observation, des stages d'initiation, d'application ou des périodes de formation en milieu professionnel [...]» (art. D331-1), même si les jeunes restent sous statut scolaire (art. D331-4). Il s'agit surtout d'une période d'observation dans le cadre de l'orientation scolaire et de la construction du projet juvénile. Les travaux portant sur l'entrée dans ce dispositif relèvent que cette option est fortement liée à la réussite scolaire et à l'origine sociale. Ce dispositif « suscite une forte adhésion très conforme aux objectifs institutionnels qui s'étaye sur des dynamiques identitaires hétérogènes liées, non pas uniquement à son utilité pour la préparation et la mise en œuvre d'un projet professionnel, mais bien davantage au rapport à l'apprendre et à la diversité des formes de l'expérience scolaire antérieures " (Bouzignac, CapdevielleMougnibas \& De Léonardis, 2015). 90 \% des élèves interrogés seraient satisfaits de cette orientation.

En même temps, la figure du maître d'apprentissage est à questionner, dans la mesure où elle ne serait pas systématiquement le support d'un tutorat « clé de voûte de la réussite pédagogique de l'alternance» (Monaco, 1995). La qualité des relations avec le maitre d'apprentissage resterait fortement liée à un travail en amont de préparation du jeune au contrat d'apprentissage, mais également d'une professionnalisation de la fonction du maître d'apprentissage, levier d'action pour accueillir l'alternant et l'accompagner pédagogiquement dans l'activité. Enfin, dans certains CFA est mise en place une forme d'accompagnement post-rupture dont l'objectif est d'abord de prospecter de nouveaux employeurs et signer un nouveau contrat d'alternance, mais qui concourt également à l'émergence de nouvelles normes du travail enseignant et à des collaborations nouvelles. En dehors même du DIMA ou de l'accompagnement post-rupture, certaines académies ont expérimenté un tutorat externe pendant le contrat d'apprentissage.

«Pendant cette expérimentation le tutorat est assuré par un conseiller de la mission locale, extérieur à l'entreprise et au centre de formation, ne dépendant ni de l'une, ni de l'autre. Il offre un appui social et professionnel basé sur l'hypothèse 
suivante : accompagner les jeunes dès le début de l'apprentissage, et tout au long du parcours, facilitera la transition de l'école à l'entreprise et évitera une rupture de contrat. Le but est de déceler au plus vite les éventuelles difficultés pour tenter d'y remédier, à la demande des jeunes, des employeurs, mais aussi des partenaires comme les centres de formation et les chambres consulaires. L'objectif est d'instaurer une politique de prévention basée sur la détection, le signalement et l'intervention rapide avec possibilité de transitions par changements d'entreprise ou d'orientation» (Bourdon et al., p. 1) ${ }^{6}$.

\section{Méthode, population et terrain}

électronique en mars 2014 sur une période de trois semaines auprès de tous les apprentisrompants d'un CFA d'Alsace (Illkirch-Graffenstaden, Communauté Urbaine de Strasbourg, Bas-Rhin), préparant aux métiers du bâtiment. Les données traitées ici sont extraites d'une recherche réalisée dans le cadre d'un mémoire de master (Hinsinger, 2014). L'échantillon regroupait les apprentis ayant rompu ou connu une rupture du contrat d'apprentissage au Centre de Formation des Apprentis entre le $1^{\text {er }}$ septembre 2010 et le 31 décembre 2013 ( $n=236)$ : 214 d'entre eux ont pu être contactés, 103 questionnaires ont été récoltés dont 82 exploitables, soit un taux de réponse de 48,1\%. Ces apprentis sont nommés des apprentis-rompants. Le centre de formation a enregistré un taux de rupture de $12 \%$ sur les trois années concernées: $30 \%$ de ces ruptures ont été suivies d'un nouveau contrat contre $70 \%$ de ruptures sèches. Rapportés aux diplômes préparés, $67 \%$ des ruptures concernaient le diplôme du Certificat d'aptitude professionnelle (CAP), 16 \% le Baccalauréat Professionnel et 7,6 \% le Brevet de Technicien Supérieur (BTS).

\section{Nos résultats}

Nous avons souhaité mettre au jour l'ensemble des variables influençant la réussite ou le décrochage des apprentis-rompants. Les résultats seront présentés en trois étapes : un tri à plat des réponses au questionnaire, une série de tableaux croisés à partir des variables significatives statistiquement (avec un seuil de signification au moins égal à 0,01 ) et une analyse factorielle des correspondances.

Dans un premier temps, l'analyse des tris à plat permet de décrire notre échantillon d'une manière monographique. Pour les 82 répondants, dont $91,5 \%$ sont des hommes, l'alternance a été à $57,3 \%$ une expérience négative. $72 \%$ ont rompu leur contrat en première année et seulement $42,7 \%$ ont bénéficié d'un accompagnement post-rupture. Les diplômes préparés étaient de niveau V à hauteur de $78 \%$ (CAP ou $\mathrm{CTM}^{7}$ ), de niveau IV (baccalauréat professionnel : 15,9\%) et de niveau III (BTS : 4,9\%). 52,4\% sont enfants uniques et $56,1 \%$ ont au moins un membre de leur famille ayant suivi auparavant une formation en apprentissage. 45,1\% déclarent avoir rencontré des difficultés personnelles pendant leur formation, d'abord dans la sphère familiale (23,2 \% des occurrences) ensuite du fait d'une maladie (7,3\%). L'origine sociale des jeunes interrogés est assez hétérogène : le père est plus fréquemment ouvrier $(20,7 \%)$, employé $(13,4 \%)$, profession intermédiaire 
$(13,4 \%)$, artisan, chef d'entreprise $(11 \%)$; rarement cadre $(4,9 \%)$ ou agriculteur $(2,4 \%)$. La mère est plus fréquemment employée $(37,8 \%)$, inactive $(17,1 \%)$, profession intermédiaire $(15,9 \%)$ et artisan, chef d'entreprise $(8,5 \%)$; rarement ouvrière $(4,9 \%)$, cadre $(2,4 \%)$ ou agricultrice $(2,4 \%)$.

Pour $45,1 \%$ des apprentis-rompants, la filière et le métier ne correspondaient pas à leurs représentations de départ. $40,2 \%$ se sont d'ailleurs réorientés pendant leur parcours d'apprenti et les raisons invoquées (mais $62,2 \%$ n'ont pas répondu à cette question) vont de l'abandon de la formation de départ au CFA (13,4\%), à la démotivation $(7,3 \%)$ en passant par l'orientation scolaire $(7,3 \%)$ et la dureté du travail (4,9\%) et concernent donc d'abord le champ scolaire de la formation initiale. $39 \%$ des rompants ont obtenu le niveau de diplôme préparé au moment de la signature du contrat d'apprentissage.

$12,2 \%$ ont bénéficié d'un DIMA avant l'apprentissage. Pour 63,4 \%, l'apprentissage était un choix d'orientation. Pour les autres $(24,4 \%)$, ils auraient souhaité intégrer la filière professionnelle en lycée et non en CFA. 19,4\% estiment qu'ils n'ont pas été acceptés en lycée professionnel du fait de leurs résultats scolaires. $70,7 \%$ des répondants avaient moins de 18 ans au début de leur formation en CFA. L'entreprise avec laquelle le contrat d'apprentissage a été signé a été contactée en premier lieu grâce au réseau familial $(45,1 \%)$, à la suite d'un stage (18,3\%), par une prospection directe du jeune $(15,9 \%)$ ou grâce à la chambre consulaire $(12,2 \%)$.

Concernant les conditions de travail, 86,6\% des apprentis-rompants ont déclaré avoir dû effectuer des heures supplémentaires, qui selon 59,8\% d'entre eux ne pouvaient être refusées. 57,3\% déclarent avoir été ponctuels, $50 \%$ assidus, 52,5\% toujours ou souvent concentrés sur leur travail. $28 \%$ seulement estiment avoir eu de mauvaises relations de travail avec leurs collègues dans l'entreprise, mais $63,4 \%$ avec le maître d'apprentissage (et $64,6 \%$ avec le chef d'entreprise qui a la plupart du temps fonction de maitre d'apprentissage). $15,9 \%$ seulement signalent des difficultés dans leurs relations avec le CFA. 24,4 \% étaient accompagnés dans leur mobilité pendulaire (contre 74,4\% qui étaient autonomes dans leurs déplacements journaliers). Pour la moitié des apprentis-rompants, se rendre dans l'entreprise représentait une difficulté en termes de déplacement. L'interlocuteur principal au CFA en cas de difficulté était le conseiller principal d'éducation $(29,3 \%)$, l'enseignant-formateur $(22 \%)$ ou un membre de la vie scolaire $(18,3 \%)$.

Au moment de la passation du questionnaire, 35,4 \% étaient au chômage, 17,1 \% encore en apprentissage, $2,4 \%$ au lycée, 9,8\% en mission d'intérim et $34,1 \%$ en contrat hors intérim.

Dans un second temps, nous avons identifié neuf corrélations statistiquement significatives dans le cadre de l'analyse de quatre phénomènes: la diplomation, la réorientation, les relations professionnelles et la qualité de l'expérience subjective $a$ posteriori des apprentis-rompants. Les non-réponses ne sont pas présentées dans les tableaux ci-dessous.

Tableau $\mathrm{n}^{\circ} 1$ : L'impact de la temporalité de la rupture sur la réorientation

\begin{tabular}{|l|l|l|l|}
\hline & Pas de réorientation & Réorientation & Total \\
\hline Rupture en $1^{\text {re }}$ année & $50,8 \%$ & $49,2 \%$ & $100 \%(59)$ \\
\hline Rupture en $2^{\text {e }}$ année & $81,8 \%$ & $18,2 \%$ & $100 \%(22)$ \\
\hline
\end{tabular}




\begin{tabular}{|l|l|l|l|}
\hline Total & $58,5 \%(48)$ & $40,2 \%(33)$ & $100 \%$ \\
\hline
\end{tabular}

$\left(X^{2}=6,37, d d l=1,1-p=98,84 \%\right)$

Alors que la corrélation entre année de rupture et diplomation n'est pas statistiquement significative $(x 2=0,09, d d l=1,1-p=23,45 \%)$, le lien entre une rupture en première année et une réorientation vers une autre filière et un autre métier est avéré (tableau $\left.n^{\circ} 1\right)$ : $49,2 \%$ des rompants de première année se réorientent contre 18,2 \% en deuxième année, soit un écart de 31 pts. Et c'est le fait de s'être réorienté qui impacte négativement la diplomation (tableau $\mathrm{n}^{\circ}$ ).

Tableau $n^{\circ} 2$ : L'impact d'une réorientation sur la diplomation

\begin{tabular}{|l|l|l|l|}
\hline & Absence de diplomation & Diplomation & Total \\
\hline Apprenti-rompant sans réorientation & $52,1 \%$ & $47,9 \%$ & $100 \%(48)$ \\
\hline Apprenti-rompant réorienté & $75,8 \%$ & $24,2 \%$ & $100 \%(33)$ \\
\hline Total & $61,0 \%(50)$ & $39,0 \%(31)$ & $100 \%$ \\
\hline
\end{tabular}

$\left(X^{2}=4,64, d d l=1,1-p=96,88 \%\right)$

Ce choix d'une réorientation dans une autre filière de formation par l'alternant après la rupture d'un contrat d'apprentissage réduit considérablement la probabilité qu'il décroche un diplôme à la fin du parcours de formation: alors que 47,9\% des apprentisrompants sans réorientation obtiennent finalement leur diplôme, ils ne sont plus que $24,2 \%$ chez ceux ayant opté pour un changement de filière d'apprentissage, soit un écart de 23,7 pts. Les apprentis réorientés sont par ailleurs légèrement surreprésentés parmi ceux ayant intégré la filière de l'apprentissage dans le cadre d'une orientation contrainte $(\chi 2=1,06, \mathrm{ddl}=1,1-\mathrm{p}=62,73 \%$, non significatif $)$.

Tableau $n^{\circ} 3$ : L'impact de la représentation de la filière sur la diplomation

\begin{tabular}{|l|l|l|l|}
\hline & Absence de diplomation & Diplomation & Total \\
\hline Filière hors représentation & $86,5 \%$ & $13,5 \%$ & $100 \%(37)$ \\
\hline Filière adaptée à la représentation & $40,9 \%$ & $59,1 \%$ & $100 \%(44)$ \\
\hline Total & $61,0 \%(50)$ & $39,0 \%(31)$ & $100 \%$ \\
\hline
\end{tabular}

$\left(X^{2}=17,67, d d l=1,1-p=99,9 \%\right)$

Lorsque l'apprenti a développé une représentation réaliste de la filière dans laquelle il s'engage, ses chances d'obtenir son diplôme malgré la rupture de contrat pendant sa formation se renforcent très significativement (tableau $n^{\circ} 3$ ) : 59,1\% des apprentisrompants qui connaissaient précisément les conditions d'exercice et les attendus du métier et de la filière avant la signature du contrat d'apprentissage ont obtenu leur diplôme contre 13,5\% des rompants s'étant engagés dans la formation sans connaissances professionnelles du métier, soit un écart de 45,6 pts. 
Tableau $n^{\circ} 4$ : L'impact des relations avec le maître d'apprentissage sur la diplomation

\begin{tabular}{|l|l|l|l|}
\hline & Absence de diplomation & Diplomation & Total \\
\hline Bonnes relations & $48,3 \%$ & $51,7 \%$ & $100 \%(29)$ \\
\hline Mauvaises relations & $69,2 \%$ & $30,8 \%$ & $100 \%(52)$ \\
\hline Total & $61,0 \%(50)$ & $39,0 \%(31)$ & $100 \%$ \\
\hline
\end{tabular}

$\left(X^{2}=3,46, d d l=1,1-p=93,71 \%\right)$

La qualité des relations interpersonnelles entre l'alternant et son maître d'apprentissage (tableau $n^{\circ} 4$ ) influence significativement la probabilité d'être ou non diplômé en fin de parcours malgré une rupture de contrat: $51,7 \%$ des répondants ayant eu de bonnes relations ont été diplômés contre $30,8 \%$ de ceux qui ont expérimenté des tensions, soit un écart de 20,9 pts.

Tableau $n^{\circ} 5$ : L'impact des représentations sur les relations avec le maître d'apprentissage

\begin{tabular}{|l|l|l|l|}
\hline & Bonnes relations & Mauvaises relations & Total \\
\hline Filière hors représentation & $21,6 \%$ & $78,4 \%$ & $100 \%(37)$ \\
\hline Filière adaptée à la représentation & $47,7 \%$ & $52,3 \%$ & $100 \%(44)$ \\
\hline Total & $35,4 \%(29)$ & $63,4 \%(52)$ & $100 \%$ \\
\hline
\end{tabular}

$\left(X^{2}=5,96, d d l=1,1-p=98,54 \%\right)$

Les relations entre l'apprenti et le maitre d'apprentissage sont d'ailleurs fortement corrélées à la connaissance que l'alternant avait en amont de la signature du contrat d'apprentissage dans cette entreprise, à la fois de la filière et du métier (tableau $\mathrm{n}^{\circ} 5$ ) : alors que $21,6 \%$ des rompants sans connaissances préalables de la réalité du métier déclarent avoir eu de bonnes relations avec le maître d'apprentissage, ils sont 47,7 \% dans le cas des apprentis avec une représentation adéquate préalable à la signature du contrat, soit un écart de 26,1 pts.

Tableau $^{\circ} 6$ : L'impact de l'accompagnement post-rupture sur la diplomation

\begin{tabular}{|l|l|l|l|}
\hline & Absence de diplomation & Diplomation & Total \\
\hline Pas d'accompagnement & $69,6 \%$ & $30,4 \%$ & $100 \%(46)$ \\
\hline Accompagnement & $51,4 \%$ & $49,6 \%$ & $100 \%(35)$ \\
\hline Total & $61,0 \%(50)$ & $39,0 \%(31)$ & $100 \%$ \\
\hline
\end{tabular}

$\left(X^{2}=2,77, d d l=1,1-p=90,38 \%\right)$

L'existence dans le parcours de l'apprenti d'un dispositif d'accompagnement post-rupture proposé par le CFA (tableau $n^{\circ} 6$ ) produit un effet positif dans la stabilisation de son parcours et ses chances d'obtenir son diplôme : 49,6 \% des rompants ayant bénéficié de ce 
dispositif ont été diplômés contre 30,4\% des rompants sans accompagnement, soit un écart de 19,2 pts.

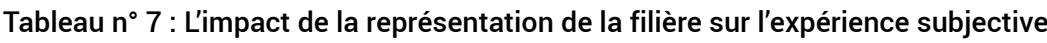

\begin{tabular}{|l|l|l|l|}
\hline & Expérience négative & Expérience positive & Total \\
\hline Filière hors représentation & $67,6 \%$ & $32,4 \%$ & $100 \%(37)$ \\
\hline Filière adaptée à la représentation & $47,7 \%$ & $50,0 \%$ & $100 \%(43)$ \\
\hline Total & $57,3 \%(46)$ & $41,5 \%(34)$ & $100 \%$ \\
\hline
\end{tabular}

$\left(x^{2}=2,86, d d l=1,1-p=90,89 \%\right)$

Très significativement, les apprentis-rompants qui expriment un ressenti négatif $a$ posteriori de leur expérience de l'alternance (tableau $\mathrm{n}^{\circ} 7$ ) sont plus fréquemment ceux pour lesquels le métier et la filière dans lesquels ils se sont engagés ne correspondaient pas à leurs représentations de départ : 67,6 \% contre $47,7 \%$, soit un écart de 19,9 pts.

Tableau $n^{\circ} 8$ : L'impact de la diplomation sur l'expérience subjective

\begin{tabular}{|l|l|l|l|}
\hline & Expérience négative & Expérience positive & Total \\
\hline Absence de diplomation & $64,0 \%$ & $34,0 \%$ & $100 \%(49)$ \\
\hline Diplomation & $46,9 \%$ & $53,1 \%$ & $100 \%(32)$ \\
\hline Total & $57,3 \%(47)$ & $41,5 \%(34)$ & $100 \%$ \\
\hline
\end{tabular}

$\left(X^{2}=2,70, d d l=1,1-p=89,96 \%\right)$

41 Logiquement, le fait que l'apprenti-rompant réussisse à obtenir son diplôme augmente la probabilité qu'il décrive son expérience de l'alternance de manière positive (tableau $\mathrm{n}$ $\left.{ }^{\circ} 8\right)$ : $53,1 \%$ des diplômés en gardent un souvenir positif contre $34,0 \%$ chez les nondiplômés, soit un écart de 19,1 pts.

Tableau $n^{\circ} 9$ : L'influence du DIMA sur les représentations des apprentis

\begin{tabular}{|l|l|l|l|}
\hline & Filière hors représentation & Filière adaptée à la représentation & Total \\
\hline Pas de DIMA & $49,3 \%$ & $50,7 \%$ & $100,0 \%(71)$ \\
\hline DIMA & $20,0 \%$ & $80,0 \%$ & $100,0 \%(10)$ \\
\hline Total & $45,1 \%(37)$ & $53,7 \%(44)$ & $100,0 \%$ \\
\hline
\end{tabular}

$\left(X^{2}=3,03, d d l=1,1-p=91,84 \%\right)$

42 Avoir suivi un DIMA (tableau $\mathrm{n}^{\circ}$ 9) exerce une influence considérable sur la manière dont les apprentis construisent leurs représentations du métier qui sont alors plus fortement en adéquation avec la réalité du champ professionnel dans lequel ils se sont engagés : $80 \%$ pour les bénéficiaires du DIMA contre $50,7 \%$, soit un écart de 29,3 pts. 

d'orientation avant l'apprentissage (par exemple, le DIMA) qui permettent au futur alternant d'acquérir une meilleure connaissance du métier et de la filière et plus tard, dans le cadre de l'accompagnement post-rupture. La temporalité de la rupture impacte, mais d'une manière indirecte la stabilité du parcours jusqu'au diplôme, dans la mesure où une réorientation précoce vers une autre filière d'alternance obère les chances de l'apprenti-rompant d'être diplômé en fin de parcours. Les relations avec le maître d'apprentissage, dans la mesure où elles sont fortement corrélées à la connaissance préalable par l'apprenti du champ professionnel visé, participent de la qualité du parcours de formation. En résumé, la préparation en amont de l'orientation juvénile, par une confrontation des représentations avec la réalité du métier, la remédiation postrupture dans le cadre d'un accompagnement individuel par le CFA et la réorientation en cours de parcours sont trois types d'action scolaire. La formation initiale apparaît ainsi comme le champ central de lutte contre le décrochage des apprentis. En même temps, la récurrence des tensions avec le maitre d'apprentissage, contrairement aux relations rarement conflictuelles avec les collègues de travail et les formateurs, interroge sur la nécessité de former les tuteurs en entreprise à la spécificité des processus d'apprentissage et des comportements juvéniles au moment de la confrontation avec l'activité.

'expérience subjective des apprentis-rompants, reconstruite après-coup, apparaît massivement négative, marquée chronologiquement par une succession de séquences: une orientation parfois contrariée (mais le regret n'est pas tant lié au désir d'intégrer la filière générale ou technologique que d'accéder au lycée professionnel), un manque de préparation documentaire sur le champ professionnel visé par le contrat d'alternance, une conflictualité avec le maitre d'apprentissage qui se confond à peu de chose près avec le chef d'entreprise ou l'artisan, une réorientation précoce vers une autre filière de l'apprentissage et une absence de diplomation. catégorisation de notre population des apprentis-rompants par le biais de l'analyse factorielle des correspondances multiples (carte factorielle ci-après). axe (en ordonnée) est celui de l'année de rupture ( $15,83 \%$ de la variance). L'analyse permet tout d'abord de scinder notre population des apprentis-rompants en deux groupes archétypiques : à gauche, les apprentis-rompants en échec n'ayant pas réussi à obtenir leur diplôme à la fin de leur apprentissage, pour lesquels l'expérience de l'alternance a été vécue négativement et dont les relations avec le maître d'apprentissage ont été mauvaises. Ces apprentis comptent parmi ceux qui n'avaient pas au préalable bénéficié d'un DIMA, qui avaient une représentation floue voire erronée de la filière dans laquelle ils se sont inscrits au moment de la signature du contrat d'apprentissage avec l'employeur. Ces jeunes n'ont pas bénéficié d'un accompagnement post-rupture et se sont réorientés dans une autre filière après la rupture, qui a eu lieu plus fréquemment lors de leur première année d'alternance. À droite, le groupe des diplômés s'oppose en tout point au premier même si les corrélations entre les modalités apparaissent plus relâchées.

Le second axe permet ensuite d'identifier deux sous-groupes spécifiques, deux séries de corrélations qui s'opposent : en haut à droite, on identifie un sous-groupe de jeunes en réussite, qui ont expérimenté une rupture salariale seulement en deuxième année, qui ne se sont pas réorientés et qui ont bénéficié d'un accompagnement post-rupture; en bas à 
gauche, on observe un sous-groupe d'apprentis dont la rupture a eu lieu en première année, qui se sont réorientés et qui n'ont bénéficié d'aucune forme d'accompagnement.

Carte factorielle : Diplomation et décrochage chez les apprentis-rompants

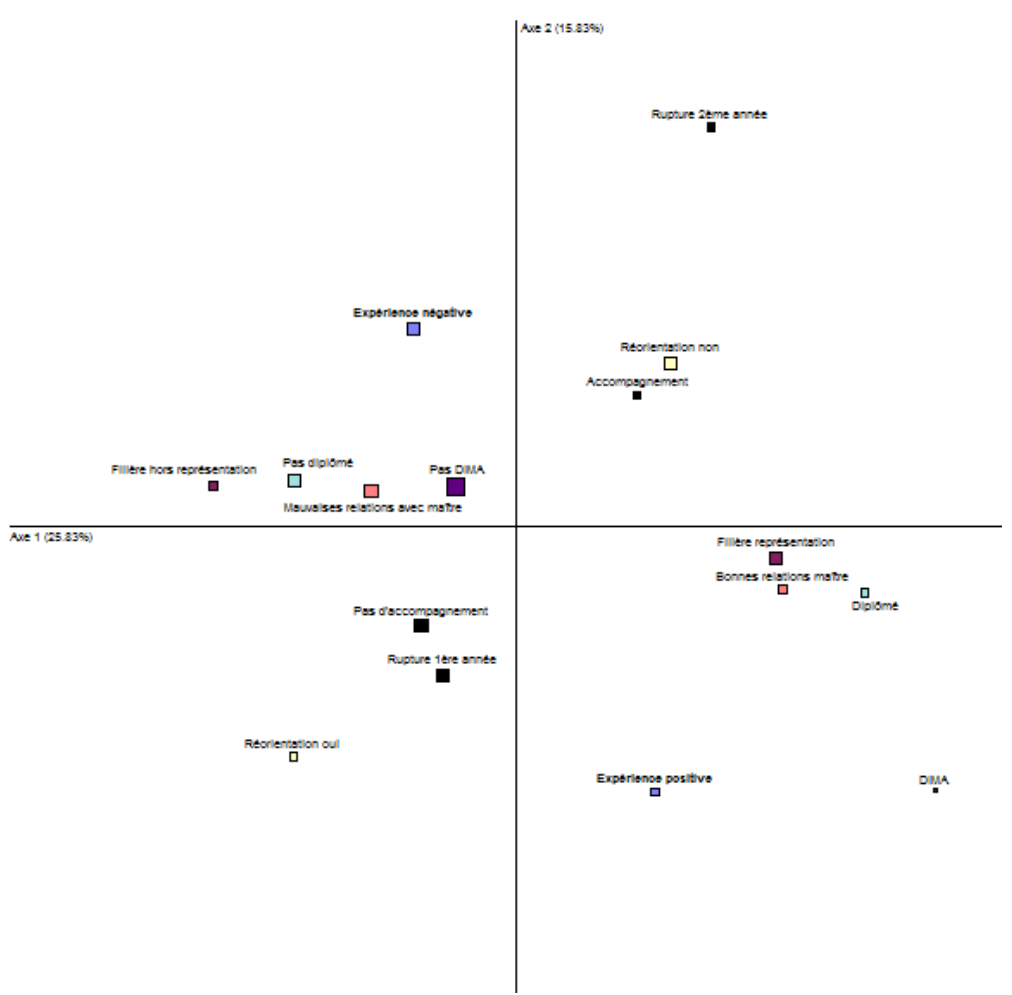

Note : La carte indique la position des 16 modalités prises en compte dans notre analyse factorielle. $41.66 \%$ de la variance est expliquée par les deux axes représentés. Les non-réponses ont été ignorées.

\section{Conclusion}

L'approche longitudinale qui a été la nôtre a inscrit le jeune alternant dans sa trajectoire d'apprentissage à l'intérieur d'un champ de socialisation double et parfois contradictoire : le centre de formation et l'entreprise : «Ouvrir la "boîte noire" du processus de rupture des contrats d'apprentissage, c'est mettre en évidence la complexité de la construction » (Mendez, 2010). Notre enquête ambitionnait d'obtenir des données utiles à la mise à plat et à l'analyse de cette complexité liée au fait qu'un phénomène de rupture peut être déterminé d'abord par le passé de l'apprenti en termes d'ancrage social, d'orientation plus ou moins affinitaire, de préparation plus ou moins aboutie au collège ; ensuite, par l'expérience directe de l'alternance dans ce qu'elle peut avoir de déstabilisant (en matière de normalisation du comportement, de régularité d'horaires, de processus cognitif, d'injonction à la productivité et à l'efficience), de conflictuel (la figure du maître d'apprentissage), voire de décevant. Nous avons vu combien la qualité des relations avec le maître d'apprentissage était fortement liée à un travail en amont de préparation du jeune au contrat d'apprentissage, relations qui participent de la viabilité et de la pérennité du projet juvénile. 
49 Dès lors qu'il est à la fois un contrat de travail et un dispositif formatif, le contrat d'apprentissage en cumule les atouts et les handicaps. Le risque de décrochage pour le jeune peut ainsi être lié au projet même, dans le cadre d'une orientation parfois hésitante ou contrariée, parfois soumise aux configurations familiales et personnelles, mais il peut être renforcé par l'environnement de travail qu'est l'entreprise d'abord en tant qu'elle est une organisation avec ses propres règles et sa culture, ensuite en tant qu'elle reste soumise aux aléas de l'activité productive. Du côté des entreprises, l'alternance peut également être une stratégie purement économique de réduction du coût du travail: certaines entreprises peuvent mésuser du contrat d'apprentissage dans l'optique d'accroître leur productivité et un tel détournement du dispositif d'alternance contribue à détériorer la qualité de l'environnement de travail de l'apprenti, expliquant parfois une rupture de contrat.

50 Le DIMA, l'accompagnement post-rupture, une absence de réorientation ou une rupture plus tardive renforcent considérablement à la fois la qualité et la stabilisation du parcours de l'alternant : comme nous l'avons vu, ces variables sont fortement interdépendantes et corrélées significativement à la qualité des relations avec le maître d'apprentissage, dans la mesure où elles signalent une adéquation forte entre un projet juvénile qui aura été conforté, documenté, outillé et une organisation économique dont la mission n'est pas tant de participer, au moment où elle l'accueille, à la construction d'un projet d'orientation que de socialiser par l'activité un jeune en cours de formation.

51 L'entreprise, cependant, dans le cadre de l'alternance, n'apparaît pas ici toujours susceptible d'anticiper voire de réduire le risque de décrochage, dans le cas où le jeune ne serait pas totalement préparé à cette entrée précoce sur le marché du travail : «Si de nombreuses actions sont mises en place pour améliorer le bien-être et éviter le décrochage des jeunes à l'entrée au lycée ou en première année de l'enseignement supérieur, elles sont plus rares pour les apprentis. Or, pour ces derniers, l'arrivée dans le monde de l'entreprise rend la transition encore plus ardue. Les interventions existantes sont morcelées, et reposent actuellement sur le suivi pédagogique des centres de formation, sur la fonction des chambres consulaires ou sur le suivi des missions locales pour les jeunes qu'elles ont orientés. Elles sont parfois tardives, davantage palliatives que préventives [...] » (Bourdon, 2012, p. 1).

Un effort de collaboration partenariale "permettant de limiter les ruptures" (Bentabet, 2012) pourrait ainsi prendre la forme d'une intensification des relations entre CFA et maîtres d'apprentissage, d'une mutualisation des savoirs et de l'expertise portant sur les spécificités juvéniles dans les processus d'apprentissage et de socialisation, avec l'idée sous-jacente de faire le départ entre ce qui dans l'entreprise est du ressort de la fonction pédagogique et d'accompagnement (le maître d'apprentissage) et de la fonction de management, de professionnalisation et d'évaluation des performances (le chef d'entreprise). Dans le même sens, des efforts de conseil et d'accompagnement en amont en direction du tissu entrepreneurial pourraient cibler plus précisément le segment secondaire du marché de l'emploi, moins efficacement structuré pour gérer la problématique des ressources humaines et la professionnalisation des fonctions d'accueil et d'accompagnement dans le cadre de l'alternance et de la gestion des emplois.

L'information et l'accompagnement s'inscrivent au cœur du processus de lutte contre le décrochage et pourraient constituer des leviers d'une prévention auprès des apprentis lorsqu'ils se destinent à l'alternance. Dans le cadre d'une formation renforcée du rôle de tuteur d'un apprenti en entreprise, l'approche de la pédagogie de l'alternance apparaît 
primordiale, combinatoire résultant d'un enchevêtrement des lieux d'apprentissage à interroger dans leurs contradictions et leur complémentarité.

\section{BIBLIOGRAPHIE}

Abriac, D., Rathelot, R., Sanchez, R. (2009). L'apprentissage entre formation et insertion professionnelles, Formations et Emploi, INSEE, 57-74.

Arrighi, J.-J., Brochier, D. (2005). 1993-2003, l'apprentissage aspiré par le haut. Bref-Céreq, nº 217.

Arrighi, J.-J., Joseph, O. (2005). L'apprentissage : une idée simple, des réalités diverses. Bref-Céreq, $n^{\circ} 223$.

Baudelot, C., \& Establet R. (2000). Avoir 30 ans en 1968 et en 1998, Paris : Le Seuil.

Bentabet, E., Cart, B., Henguelle, V., \& Toutin, M.-H. (2012). Jeunes et entreprises face aux ruptures de contrat d'apprentissage. Étude sur les effets des ruptures de contrat d'apprentissage sur les trajectoires des jeunes et sur les pratiques des entreprises, Clersé, Céreq, Rapport CPC études $\mathrm{n}^{\circ} 1$, ministère de l'Éducation nationale, Direction générale de l'enseignement scolaire.

Boléguin, V. (2011). La Réunion : une jeunesse tiraillée entre tradition et modernité. Les 16-30 ans au chômage, Paris : L'Harmattan.

Bourdon, J., Guégnard, C., \& Michot, C. (2012). Sécuriser les parcours des apprentis. Bref-Céreq, n - 301.

Bouzignac, V., Capdevielle-Mougnibas, V., \& De Léonardis, M. (2015). Projet professionnel, rapport à l'apprendre et sens de l'expérience scolaire chez des collégien(ne)s orienté(e)s vers le Dispositif d'Initiation aux Métiers de l'Alternance (DIMA). L'orientation scolaire et professionnelle, $44 / 2$.

Carré, P. (1999). Motivation et rapport à la formation. In P. Carré \& P. Caspar, Traité des sciences et des techniques de la Formation, Paris : Dunod.

Cart, B., Ducourant, H., Henguelle, V., Surelle, A.-S., \& Toutin M.-H. (2007). Les ruptures des contrats d'apprentissage en Région Nord-Pas de Calais. C2RP, OREF Nord.

Cart, B., Toutin Trelcat, M.-H., \& Henguelle, V. (2010). Contrat d'apprentissage, les raisons de la rupture. Bref-Céreq, $\mathrm{n}^{\circ} 272$.

CÉREQ (2011). Présentation des premiers résultats de l'enquête génération. 2007-2010. Premiers pas dans la vie active : Le diplôme : un atout gagnant pour les jeunes face à la crise. Enquête Génération du Céreq. Ministère de l'Éducation nationale.

Dréano, R. (2002). Qualité de l'apprentissage : conditions de vie des apprentis. Rapport à la Secrétaire d'État aux Droits des Femmes et à la Formation Professionnelle, Paris : La Documentation française.

EFIGIP (2010). Ruptures des contrats en alternance. Contrats d'apprentissage et de professionnalisation jeune. Emploi Formation Insertion en Franche-Comté.

Geay, A. (1998). L'école de l'alternance. Paris : L'Harmattan. 
Hinsinger, S. (2014). La rupture du contrat d'apprentissage : un processus conflictuel aux multiples facteurs. Mémoire de Master en Sciences de l'Éducation, dir. S. Guillon, Université de Strasbourg. Kergoat, P., \& Capdevielle-Mougnibas, V. (2013). Les formations par apprentissage : un domaine de recherche à développer. Revue française de pédagogie, $\mathrm{n}^{\circ}$ 183, 5-14.

Lamamra, N., \& Masdonati, J. (2008). Adolescence en souffrance : stratégies des jeunes face aux contraintes de la formation professionnelle. Reflets. Revue d'intervention sociale et communautaire, $\mathrm{n}$ • 14, 67-102.

Longhi, G. (2011). Pour une persévérance scolaire généralisée. La nouvelle revue de l'adaptation et de la scolarisation, $\mathrm{n}^{\circ}$ 56, 179-191.

Masdonati, J., Lamamra, N. (2007). La formation professionnelle entre continuités et ruptures : témoignages de jeunes en situation de décrochage. $3^{\mathrm{e}}$ rencontres Jeunes \& sociétés en Europe et autour de la Méditerranée. Marseille.

Masdonati, J. (2007). La transition entre l'école et le monde du travail. Bern : Peter Lang.

Mauger, G. (2001). Les politiques d'insertion, une contribution paradoxale à la déstabilisation du marché du travail. Actes de la recherche en sciences sociales, $\mathrm{n}^{\circ}$ 136-137, 5-14.

Mendez, A. (dir.) (2010). Processus : concepts et méthodes pour l'analyse temporelle en sciences sociales, Louvain-la-Neuve : Academia Brylant.

Michaud, P.-A. (2001). Prévenir les ruptures, limiter leurs conséquences. Panorama, n 6, 8-10.

Monaco, A. (1995). Le statut du tutorat en entreprise. Paris : CRDP.

Moreau, G. (2002) (coord.). Les patrons, l'État et la formation des jeunes. Paris : La Dispute.

Moreau, G. (2003). Le monde apprenti. Paris : La Dispute.

Moreau, G. (2008). Apprentissage : une singulière métamorphose. Formation emploi, $\mathrm{n}^{\circ} 101$, 119-133.

Ramé, S., \& Ramé, L. (1995). La formation professionnelle par apprentissage. État des lieux et enjeux sociaux. Paris : L'Harmattan.

Rochat S., \& Lamamra N. (2004). La socialisation des apprentis. Panorama, n 4, 21-22.

Rongé, J.-L. (2011). Apprentissage et formation en alternance : le travail à 14 ans. Association Jeunesse et Droit. Journal du Droit des Jeunes, $\mathrm{n}^{\circ}$ 308, 45-47.

Rose, J. (1998). Les jeunes face à l'emploi. Paris : Desclée de Brouwer.

Tanguy, L. (2005). De l'éducation à la formation : quelles réformes ? Éducation et Sociétés, $\mathrm{n}^{\circ} 16$, 99-122.

Tanguy, L. (2013). Apprentissage en entreprise et formation professionnelle en école : une mise en perspective des années 1950 aux années 1990. Revue française de pédagogie, n 183, 27-38.

\section{NOTES}

1. Loi $n^{\circ} 71-576$, dite loi «Guichard » qui prévoit la création des CFA, la tutelle de l'Éducation nationale et l'encadrement du contrat d'apprentissage.

2. Cette loi $n^{\circ} 87-572$ du 23 juillet 1987 ouvre l'apprentissage à l'ensemble des diplômes professionnels et techniques, y compris dans le supérieur, et reporte à 25 ans l'âge limite pour la signature du contrat. La loi «Cresson » de 1992 ouvrira l'apprentissage aux diplômes d'ingénieur. 
3. L'employeur qui embauche un apprenti bénéficie d'une exonération de cotisations sociales patronales, d'une prime à l'apprentissage et d'un crédit d'impôt (Loi n²013-1278 du 29 décembre 2013).

4. Aujourd'hui, de nombreuses régions permettent à un apprenti-rompant de poursuivre sa formation au CFA sur quelques mois, le temps pour lui de retrouver une entreprise ou pour passer les examens éventuels.

5. Dans le Code de l'Éducation, trois textes traitent de ce dispositif : les articles D337-172 à D337-182 portant sur le fonctionnement du dispositif ; l'article L337-3-1 sur les conditions pour en bénéficier et les articles D331-1 à D331-15 sur le déroulement de la formation. Le DIMA remplace depuis la rentrée 2008 la Classe Préparatoire à l'Apprentissage (CPA) créée par la circulaire $n^{\circ} 72-109$ du 10 mars 1972 et en reprend les grandes lignes.

6. Au moment de notre enquête, en région Alsace, une enveloppe budgétaire régionale avait été allouée à un suivi des jeunes alternants (âgés d'au moins 16 ans) pour prévenir la rupture. Cette expérimentation avait d'abord eu lieu au sein des Apprentis d'Auteuil avant d'être élargie à certains CFA qui en exprimaient le besoin.

7. Le Certificat Technique des Métiers, titre de niveau V, est délivré par les Chambres de Métiers, uniquement par la voie de l'apprentissage, après deux ou trois ans, accessible après la classe de troisième.

\section{RÉSUMÉS}

La rupture du contrat de travail dans le cadre d'un apprentissage renforce le risque du décrochage scolaire, en tout cas d'une précarisation du parcours de formation du jeune et d'une réduction de ses chances d'obtenir le diplôme préparé. Pourtant, des dispositifs en amont et en aval de l'apprentissage permettent d'augmenter les chances de réussite malgré cette rupture du lien salarial dont les causes ne sont pas majoritairement à chercher dans les conditions de travail, les tâches à réaliser ou le savoir-être juvénile. Notre article présente les résultats d'une enquête par questionnaire réalisée en 2014 auprès de 214 apprentis ayant rompu leur contrat entre 2010 et 2013, inscrits dans un Centre de Formation d'Apprentis (CFA) en Alsace. Le décrochage peut être évité par une préparation à l'alternance, mais également par un accompagnement après la rupture, qui améliorent à la fois l'orientation scolaire, les relations professionnelles avec le maitre d'apprentissage, réduisent le phénomène de réorientation en première année et augmentent les chances de diplomation.

The breach of contract during an apprenticeship strengthens the risk of dropout, weakens the training period and reduces chances for graduation. Nonetheless some pedagogical measures taken before and after an apprenticeship can make success possible despite the breach of contract, which most of the time is not due either to work conditions or professional tasks nor apprentices' motivation. Our paper presents some results of research made in 2014 questioning 214 apprentices who have faced a contract breach in apprenticeship between 2010 and 2013, enrolled in a training center in Alsace. We show that dropout can be avoided if the apprenticeship period had been prepared but also if the apprentice is accompanied after the breach. Both measures improve school orientation, professional relationship with the apprentice's master, make reorientation less frequent and above all enable further graduation. 
INDEX

Mots-clés : alternance, apprentissage, décrochage, parcours, rupture de contrat

Keywords : apprenticeship, learning, dropout, schooling, breach of contract

\section{AUTEURS}

\section{STÉPHANE GUILLON}

Maître de conférences, LISEC, EA 2310, Faculté des Sciences de l'Éducation, Université de Strasbourg

\section{STÉPHANIE HINSINGER}

Coordinatrice pédagogique, École Régionale de la Deuxième Chance, Narbonne 